\title{
PREVENÇÃO DO ESCURECIMENTO DO PERICARPO DE LICHIA ATRAVÉS DO USO DE ÁCIDOS E FILMES ${ }^{1}$
}

\author{
DANIELLE FABÍOLA PEREIRA DA SILVA², LUIZ CARLOS CHAMHUM SALOMÃO, \\ ELAINE CRISTINA CABRINI ${ }^{4}$, ROBSON RIBEIRO ALVES ${ }^{5}$, TIAGO BARBOSA STRUIVING ${ }^{6}$
}

RESUMO - Neste trabalho, foram avaliados os efeitos de ácidos e filmes na prevenção do escurecimento do pericarpo de lichia. Lichias 'Bengal' foram colhidas com o pericarpo completamente vermelho e submetidas aos seguintes tratamentos: imersão em ácido cítrico $100 \mathrm{mM}$, por 5 minutos; imersão em ácido clorídrico $(\mathrm{HCl}) 1 \mathrm{~N}$, por 2 minutos; imersão em fécula de mandioca $30 \mathrm{~g} / \mathrm{L}$, por 5 minutos, e filme de policloreto de vinila (PVC) de $14 \mu \mathrm{m}$ de espessura, além do controle, não tratado.Os frutos de todos os tratamentos foram acondicionados em bandejas de poliestireno $(220 \mathrm{~mm}$ x $140 \mathrm{~mm}$ x $40 \mathrm{~mm})$, armazenados em câmara fria a $5 \pm 1,2^{\circ} \mathrm{C}$ e $90 \pm 5 \%$ de UR e avaliados a cada 4 dias, durante 20 dias. A fécula de mandioca e o ácido cítrico não foram eficientes em prevenir ou retardar o escurecimento do pericarpo da lichia. O filme de PVC manteve a coloração vermelha do pericarpo somente até o quarto dia de armazenamento. $\mathrm{O} \mathrm{HCl}$ foi efetivo em prevenir o escurecimento do pericarpo dos frutos durante todo o período experimental sem prejudicar a qualidade pós-colheita.

Termos para indexação: Litchi chinensis Sonn., polifenoloxidase, peroxidase, qualidade pós-colheita.

\section{PREVENTION OF PERICARP BROWNING OF LYCHEE THROUGH THE USE OF ACIDS AND FILMS}

\begin{abstract}
The effects of acids and films in preventing the pericarp browning of lychee were evaluated. 'Bengal' lychees with pericarp completely red were harvested and treated as follows: immersion in citric acid $100 \mathrm{mM}$ for 5 minutes, immersion in hydrochloric acid $1 \mathrm{~N}$ for 2 minutes, immersion in cassava starch $30 \mathrm{~g} / \mathrm{L}$ for 5 minutes and the film of polyvinyl chloride (PVC) $14 \mathrm{~mm}$ thick, and a control, untreated. The fruits of all treatments were placed in polystyrene trays $(220 \mathrm{~mm} \times 140 \mathrm{~mm} \times 40 \mathrm{~mm})$, stored in a chamber at $5.0 \pm 1.2^{\circ} \mathrm{C}$ and $90 \pm 5 \% \mathrm{UR}$, and were evaluated every 4 days, for 20 days. The cassava starch and citric acid were not effective in preventing or delaying the browning of the lychee pericarp. The PVC maintained the red color of the pericarp only until the fourth day of storage. The $\mathrm{HCl}$ was effective in preventing the pericarp browning throughout the experimental period without spoiling the post-harvest quality.
\end{abstract}

Index terms: Litchi chinensis Sonn., polyphenoloxidase, peroxidase, post-harvest quality

\footnotetext{
${ }^{1}$ Trabalho Sinfruit 063 - Simpósio Internacional de Fruticultura - Avanços na Fruticultura (17 a 21 Outubro)

${ }^{2}$ Eng. Agr ${ }^{\mathrm{a}}$. DSc. Pós-Doutoranda (Bolsista PNPD/CAPES) - Departamento de Fitotecnia - Universidade Federal de Viçosa - CEP 36.570-000 - Viçosa-MG - Brasil - danieele@ufv.br;

${ }^{3}$ Eng. Agr. DSc. - Prof. Departamento de Fitotecnia - Universidade Federal de Viçosa - CEP 36.570-000 - Viçosa-MG- Brasil - (Bolsista Produtividade CNPq) - 1salomao@ufv.br

${ }^{4}$ Biológa- DSc. - Prof ${ }^{\mathrm{a}}$ - Universidade Federal Vales Jequitinhonha e Mucuri - Campus Universitário II- Diamantina-MG-elaineccabrini@hotmail.com

${ }^{5}$ Químico - Estudante do Curso de Doutorado - Departamento de Química - Universidade Federal de Viçosa - CEP 36.570-000 Viçosa-MG - Brasil - robson@ufv.br

${ }^{6}$ Estudante do Curso de Agronomia - Departamento de Fitotecnia - Universidade Federal de Viçosa - CEP 36.570-000 - Viçosa-MGBrasil - (Bolsista CNPq) - struiving@hotmail.com
} 


\section{INTRODUÇ̃̃O}

O escurecimento do pericarpo da lichia é um dos maiores entraves à comercialização deste fruto. Mesmo causando pouca alteração na qualidade da polpa, o escurecimento prejudica a aparência do fruto, requisito de qualidade fundamental na aquisição de um produto pelo consumidor (MIZOBUTSI et al., 2010).

A rápida mudança da coloração vermelha para uma tonalidade marrom é verificada inclusive em frutos ainda ligados à planta. Em frutos colhidos, este escurecimento pode ocorrer em poucas horas ou, no máximo, três dias após a colheita em temperatura variando de $20^{\circ}$ a $25^{\circ} \mathrm{C}$ (HOJO et al., 2011).

Os frutos deterioram-se rapidamente, a menos que sejam empregadas técnicas adequadas de conservação. $\mathrm{O}$ armazenamento à temperatura entre 1 e $5^{\circ} \mathrm{C}$ é usado para reduzir as doenças pós-colheita, mas estas temperaturas não evitam o escurecimento do pericarpo. Além disso, os frutos deterioram-se rapidamente após o armazenamento por aproximadamente 30 dias (REUCK et al., 2011).

De acordo com Del Aguila et al. (2009), a utilização do frio na pós-colheita de frutos é uma tecnologia indispensável quando o objetivo é a conservação e a manutenção da qualidade destes frutos. Entretanto, é necessária a utilização de outras tecnologias complementares ao frio, com as quais haverá maiores possibilidades de se manter a qualidade do produto após a colheita. Dentre as técnicas complementares ao frio, tem-se a utilização de atmosfera modificada através de filmes plásticos ou biofilmes gerados, por exemplo, pela fécula de mandioca.

Diversos tratamentos foram realizados visando à redução do escurecimento em lichia, principalmente a imersão em ácido ascórbico, ácido cítrico, lecitina, ceras, tratamentos com compostos à base de enxofre e acondicionamento em embalagens plásticas (SILVA et al., 2010; DUCAMP-COLLIN et al., 2008). Já a redução da perda de umidade é obtida com o revestimento dos frutos com filmes plásticos, a exemplo do polipropileno (PP) (REUCK et al., 2011).

Desta forma, o objetivo deste trabalho foi avaliar o uso de ácidos e filmes na prevenção do escurecimento do pericarpo de lichia.

\section{MATERIAL E MÉTODOS}

Frutos de lichieira (Litchi chinensis Sonn.) 'Bengal' com pericarpo completamente vermelho foram colhidos no período da manhã, em dezembro de 2007, de plantas do Pomar Experimental da
Universidade Federal de Viçosa, em Viçosa-MG ( $21^{\circ} 07^{\prime} \mathrm{S}, 42^{\circ} 57^{\prime} \mathrm{W}, 651 \mathrm{~m}$ de altitude).

Após a colheita, foram selecionados os frutos com pericarpo uniformemente vermelho. Estes foram imersos em fungicida Prochloraz (Sportak $450 \mathrm{CE}$, Hoechst Schering AgrEvo UK Ltd., Inglaterra), na dose de 49,5 g/100 L de água, por 5 minutos, e secos à temperatura ambiente.

Subsequentemente, foram submetidos aos seguintes tratamentos: imersão em ácido cítrico 100 $\mathrm{mM}$, por 5 minutos; imersão em ácido clorídrico ( $\mathrm{HCl}) 1 \mathrm{Nl}$ por 2 minutos; imersão em fécula de mandioca $30 \mathrm{~g} / \mathrm{L}$, por 5 minutos, e filme de policloreto de vinila (PVC) de $14 \mu \mathrm{m}$ de espessura, além do controle não tratado. Os frutos de todos os tratamentos foram acondicionados em bandejas de poliestireno $(220 \mathrm{~mm}$ x $140 \mathrm{~mm}$ x $40 \mathrm{~mm}$ ), armazenados em câmara fria a $5 \pm 1,2^{\circ} \mathrm{C}$ e $90 \pm 5 \%$ de UR e avaliados a cada 4 dias, durante 20 dias.

Foram avaliados perda de massa fresca, atributos de cor do pericarpo, $\mathrm{pH}$, teor de sólidos solúveis e acidez titulável da polpa, vitamina $\mathrm{C}$ do pericarpo e da polpa e atividades da polifenoloxidase (PPO) e peroxidase (POD) do pericarpo. A perda de massa fresca foi determinada por gravimetria, sendo os resultados expressos em porcentagem de perda de massa. Para a determinação do $\mathrm{pH}$ da polpa, foram feitas leituras de amostras do tecido, em pHmetro digital, após trituração em liquidificador.

A coloração do pericarpo foi determinada por reflectometria, utilizando-se de um reflectômetro Minolta (Color Reader CR- 10). Foram feitas duas leituras por fruto em posições diametralmente opostas. As coordenadas utilizadas foram o L* (luminosidade), que varia de 0 (preto) a 100 (branco); e a*, que varia do verde $(-60)$ ao vermelho $(+60)$ (McGUIRRE, 1992).

Para a determinação das atividades da polifenoloxidase (PPO) e peroxidase (POD) do pericarpo, foram utilizados os métodos descritos por Flurkey e Jen (1978) e Maia et al. (2008), com modificações. Para a obtenção do extrato enzimático, $1 \mathrm{~g}$ do pericarpo, com $5 \mathrm{~mL}$ de tampão fosfato $0,2 \mathrm{M}(\mathrm{pH}=$ $6,0)$, resfriado em banho de gelo, foi triturado em politron a $20.500 \mathrm{rpm}$, por $40 \mathrm{~s}$. A suspensão foi centrifugada a $10.000 \mathrm{~g}$, por 21 minutos, a $4{ }^{\circ} \mathrm{C}$. Para a determinação da atividade, foram efetuadas sete leituras de absorvância de 30 em 30 segundos, em espectrofotômetro a $\lambda=425 \mathrm{~nm}$ (PPO) e $\lambda=470 \mathrm{~nm}$ (POD). Os resultados foram expressos em unidade da enzima por grama de amostra, calculada a partir da quantidade de extrato, que acusou um aumento na absorvância de 0,01 unidades por minuto.

A vitamina $\mathrm{C}$ do pericarpo e da polpa foi de- 
terminada por titulação com reagente de Tillman [2,6 diclorofenolindofenol (sal sódico) a 0,1\%] (AOAC, 1997). Os resultados foram expressos em mg 100 $\mathrm{g}^{-1}$ de amostra. O teor de sólidos solúveis da polpa foi determinado com o auxílio de um refratômetro digital, em amostras de polpa trituradas em liquidificador (AOAC, 1997). A acidez titulável da polpa foi determinada por titulação com $\mathrm{NaOH} 0,1 \mathrm{~N}$ e expressa em porcentagem de ácido málico (AOAC, 1997).

O experimento foi conduzido em parcelas subdivididas, tendo-se nas parcelas os cinco tratamentos e, nas subparcelas, os seis períodos de amostragens, sendo a unidade experimental constituída por seis frutos. $\mathrm{O}$ delineamento experimental foi o inteiramente casualizado, com três repetições. Os dados foram analisados por meio das análises de variância e regressão, usando-se o programa SAEG 9.1 - Sistema para Análises Estatísticas e Genéticas. Para os dados de atividade das enzimas polifenoloxidase e peroxidase, utilizaram-se análises descritivas.

\section{RESULTADOS E DISCUSSÃO}

A perda de massa fresca aumentou linearmente com o incremento do período de armazenamento (Figura 1A). A perda registrada no $20^{\circ}$ dia de armazenamento foi de 13,2; 12,99; 13,88; 16,44 e $3,12 \%$ para os tratamentos-controle, $\mathrm{HCl}$, ácido cítrico, fécula e filme, respectivamente. Apesar de efetivo em outros frutos, o uso de fécula não trouxe benefícios na redução da perda de umidade ou do escurecimento do pericarpo de lichias. Segundo Lima et al. (2010), a descoloração ocorre geralmente logo após a aplicação do biofilme.

Resultados similares de perda de massa fresca (ao redor de 13\%) foram obtidos por Nagar (1994), em lichia 'Calcuttia', ao longo de 10 dias de armazenamento a $25^{\circ}$ e $80 \%$ de UR. Perdas de massa fresca superiores a $6,6 \%$ resultam em escurecimento o pericarpo das lichias, como observado nos frutos dos tratamentos-controle, fécula e ácido cítrico, a partir do $8^{0}$ dia de armazenamento; já o escurecimento total do pericarpo é observado com perdas de massa fresca superiores a 18,21\% (CHEN et al., 2001). Entretanto, para Mahajan e Goswami (2004), as perdas de massa fresca que causam escurecimento no pericarpo destes frutos são menores, na faixa de 3 a $5 \%$.

A luminosidade $\left(\mathrm{L}^{*}\right)$ do pericarpo, nos cinco tratamentos avaliados, apresentou diferenças significativas ao longo do armazenamento, mostrando tendência de queda (Figura 1B). As reduções no $20^{\circ}$ dia de armazenamento, em relação ao dia do armazenamento, foram bruscas para os tratamentos- -controle (58,16\%), ácido cítrico (41,21\%), fécula $(41,21 \%)$ e filme $(62,81 \%)$. Todavia, observa-se que, para o tratamento $\mathrm{HCl}$, a redução de $\mathrm{L}^{*}$ foi de apenas $6,39 \%$, o que demonstra o potencial deste ácido em manter a coloração do pericarpo da lichia. A imersão dos frutos em soluções diluídas de $\mathrm{HCl}$ tem possibilitado o restabelecimento da coloração vermelha pela conversão de pigmentos escuros a íons flavino, que são predominantes em $\mathrm{pH}$ baixo. Zauberman et al. (1991) demonstraram que a imersão dos frutos em $\mathrm{HCl}$ a $1 \mathrm{~N}$ por dois minutos resultou em completa recuperação da coloração dentro de 24 a 48 horas após o tratamento.

Observou-se tendência a redução para a coordenada $a^{*}$ (Figura 1C). As maiores reduções de $a^{*}$ foram observadas para os tratamentos-controle, ácido cítrico e fécula. Todavia, para os tratamentos filme e $\mathrm{HCl}$, os valores de $\mathrm{a}^{*}$ mantiveram-se praticamente constantes, confirmando a manutenção da cor vermelha principalmente nos frutos tratados com $\mathrm{HCl}$. A mudança indesejável da coloração do pericarpo da lichia, principalmente com um rápido decréscimo da luminosidade $\left(\mathrm{L}^{*}\right)$, unido a um decréscimo dos valores iniciais de $\mathrm{a}^{*}$, dão como resultado principal escurecimento e perda da coloração vermelha intensa do pericarpo, cuja consequência imediata é a perda de seu valor comercial ou uma forte redução do preço de comercialização destes frutos no varejo.

A rápida alteração da coloração vermelha para uma tonalidade marrom Também ocorre em frutos ainda ligados à planta. Em frutos colhidos, este escurecimento pode ocorrer em poucas horas ou, no máximo, três dias após a colheita, em temperatura variando de $20^{\circ} \mathrm{a} 25^{\circ} \mathrm{C}$ (LIMA et al., 2010).

Observou-se pouca variação nos valores de pH da polpa ao longo do período experimental (Figura $2 \mathrm{~A}$ ), tendo os valores médios entre os tratamentos ficado entre 4,2 no dia zero e 4,7 no $20^{\circ}$ dia de armazenamento. Acréscimos do $\mathrm{pH}(4,58$ e 5,03$)$ da polpa de lichia 'Bengal' armazenadas a $5^{\circ} \mathrm{C}$ também foram observados por Del Aguila et al. (2009).

Houve redução no teor de sólidos solúveis nos frutos de todos os tratamentos durante o armazenamento, embora com menor intensidade nos tratados com $\mathrm{HCl}$ (Figura 2B). Esse comportamento deve refletir o consumo de carboidratos durante o processo respiratório. De forma similar, Nagar (1994) obteve redução dos teores de SST em lichia 'Calcuttia' ao longo de 10 dias de armazenamento a $25^{\circ} \mathrm{C}$ e $80 \%$ de UR.

Foram observadas reduções superiores a 50\% nos teores de acidez titulável dos frutos, nos diferentes tratamentos, no decorrer do período experimental (Figura 2C). Resultados semelhantes foram encon- 
trados por Nagar (1994), que obteve decréscimo da ordem de 50\% na acidez das lichias 'Calcuttia' ao longo de 10 dias de armazenamento a $25^{\circ} \mathrm{C}$ e $80 \%$ de UR.

A vitamina $\mathrm{C}$ do pericarpo teve queda brusca para os tratamentos-controle, ácido cítrico, fécula e filme (Figura 3A). No dia zero, o teor de vitamina $\mathrm{C}$, em todos os tratamentos, era em torno de 109 $\mathrm{mg}$ ácido ascórbico. $100^{-1} \mathrm{~g}$ de pericarpo; no oitavo dia de armazenamento, estes teores eram de 24,21; 5,$72 ; 20,26 ; 2,88$ e $96,90 \mathrm{mg}$, respectivamente, demonstrando que o $\mathrm{HCl}$ é efetivo não só em manter a coloração vermelha do pericarpo, como também em manter alto os teores de vitamina $\mathrm{C}$ do pericarpo. $\mathrm{O}$ efeito benéfico da manutenção dos teores de ácido ascórbico no pericarpo atribui-se a vários aspectos, sendo um destes o efeito de captação do oxigênio e proteção, formando uma barreira que impede a difusão do oxigênio para o interior do produto, reduzindo as quinonas geradas e inibindo a ação das PPos (REUCK et al., 2011), com consequente manutenção da coloração vermelha.

Verificaram-se, de maneira geral, decréscimos nos teores de ácido ascórbico da polpa dos frutos dos diferentes tratamentos (Figura 3B). As perdas registradas foram de 98,55; 99,28; 92,25; 93,09 e 71,64\% para os tratamentos ácido cítrico, filme, controle, fécula e HCl, respectivamente. Del Aguila et al. (2009), trabalhando com lichias 'Bengal' armazenadas a $5^{\circ} \mathrm{C}$, observaram decréscimos de $67,26 \%$ nos teores de vitamina $\mathrm{C}$, no $15^{\circ}$ dia de armazenamento.

Os ácidos, além dos açúcares, constituem substratos para a respiração, sendo que o ácido ascórbico participa de reações antioxidantes que se processam durante a maturação e após a colheita do fruto (NEVES et al.; 2011). Possivelmente, devido ao estresse da colheita, ocorra no fruto maior atividade metabólica, principalmente maior taxa respiratória, havendo consumo de ácidos nesse processo (WATANABE et al., 2011). Além disso, a utilização de ácido ascórbico em reações regenerativas, principalmente próximas às membranas celulares, na intenção de manter a permeabilidade seletiva das membranas celulares e, por consequência, adiar a morte programada, pode ocasionar diminuição nos teores de ácido ascórbico (DÍAZ-MULA et al., 2009).

A atividade enzimática das peroxidases foi máxima no quarto dia de armazenamento (Figura 4A), sendo as menores atividades registradas para os tratamentos PVC e $\mathrm{HCl}$. A maior atividade enzimática foi do tratamento fécula, esse fato pode ser explicado pelo maior dessecamento do pericarpo ocorrido nos frutos deste tratamento. Resultados semelhantes foram encontrados por Zhang et al. (2004), que observaram alta atividade da peroxidase $\left(8,5 \times 10^{3} \mathrm{U} \cdot \mathrm{min}^{-1} \cdot \mathrm{g}^{-1} \mathrm{MF}\right)$ para lichias $\mathrm{cv}$. Huaizhi, no quarto dia de armazenamento, à temperatura de $28^{\circ} \mathrm{C}$.

Os frutos revestidos com fécula de mandioca apresentaram maior atividade da polifenoloxidase (Figura 4B), enquanto a menor atividade enzimática foi verificada no tratamento com $\mathrm{HCl}$, seguida do filme de PVC.

O escurecimento do pericarpo da lichia é causado pela oxidação dos substratos fenólicos catalisados pela polifenoloxidase (PPO) (DUCAMP-COLLIN et al., 2008). Em tecidos intactos, a PPO está separada de seus substratos devido à compartimentação das membranas. A perda da integridade da membrana permite o contato da enzima com seu substrato, iniciando a reação de escurecimento enzimático. Uma perda rápida da integridade da membrana da lichia foi verificada após a colheita, ao obter-se aumento do vazamento de eletrólitos (JIANG; FU, 1999). 

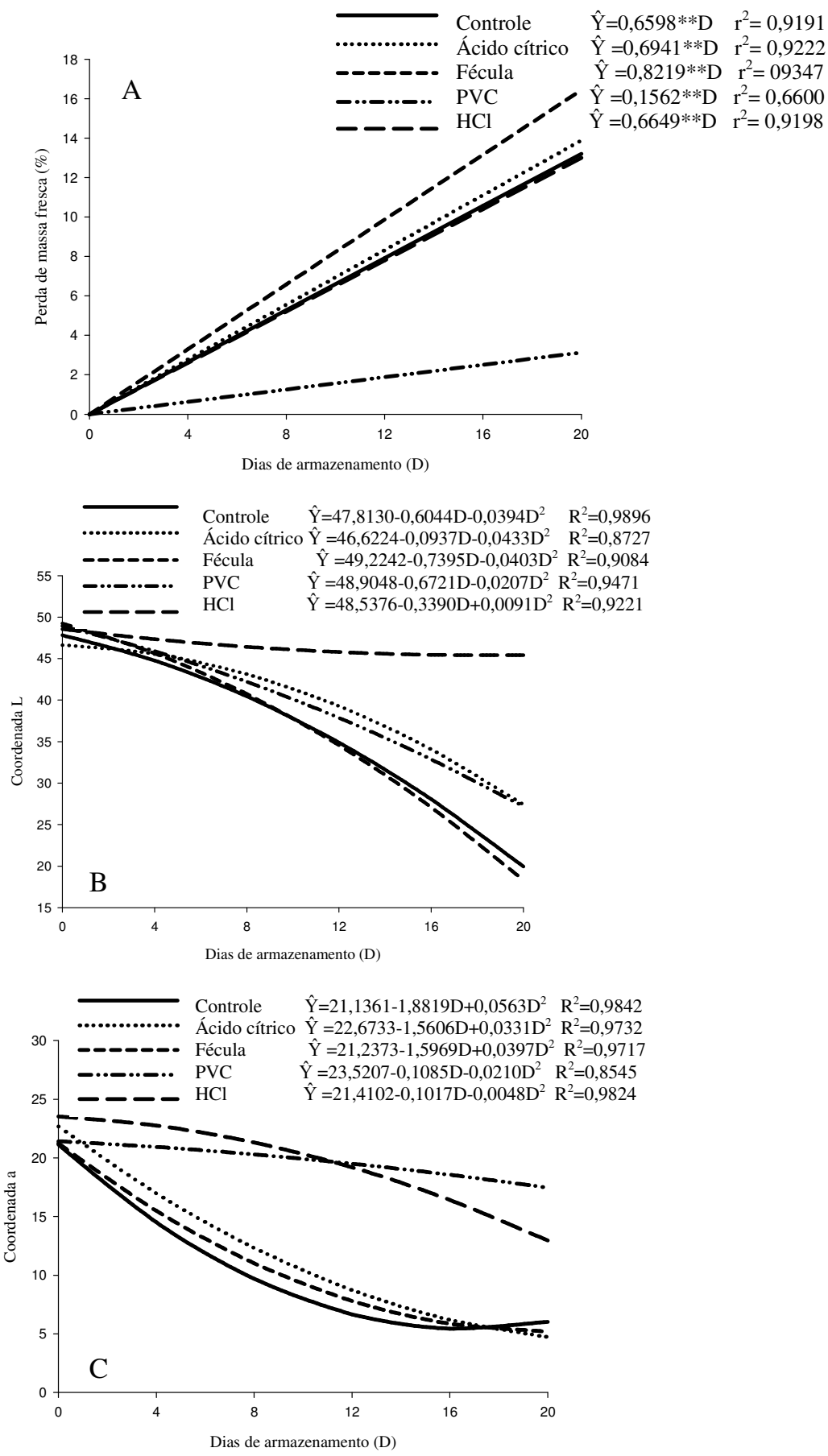

FIGURA 1- Perda de massa fresca (\%) (A); coordenada L* (B) e coordenada a* (C) de frutos de lichieira 'Bengal' armazenados a $5 \pm 1,2{ }^{\circ} \mathrm{C}$ e $90 \pm 5 \%$ de UR, por 20 dias. 

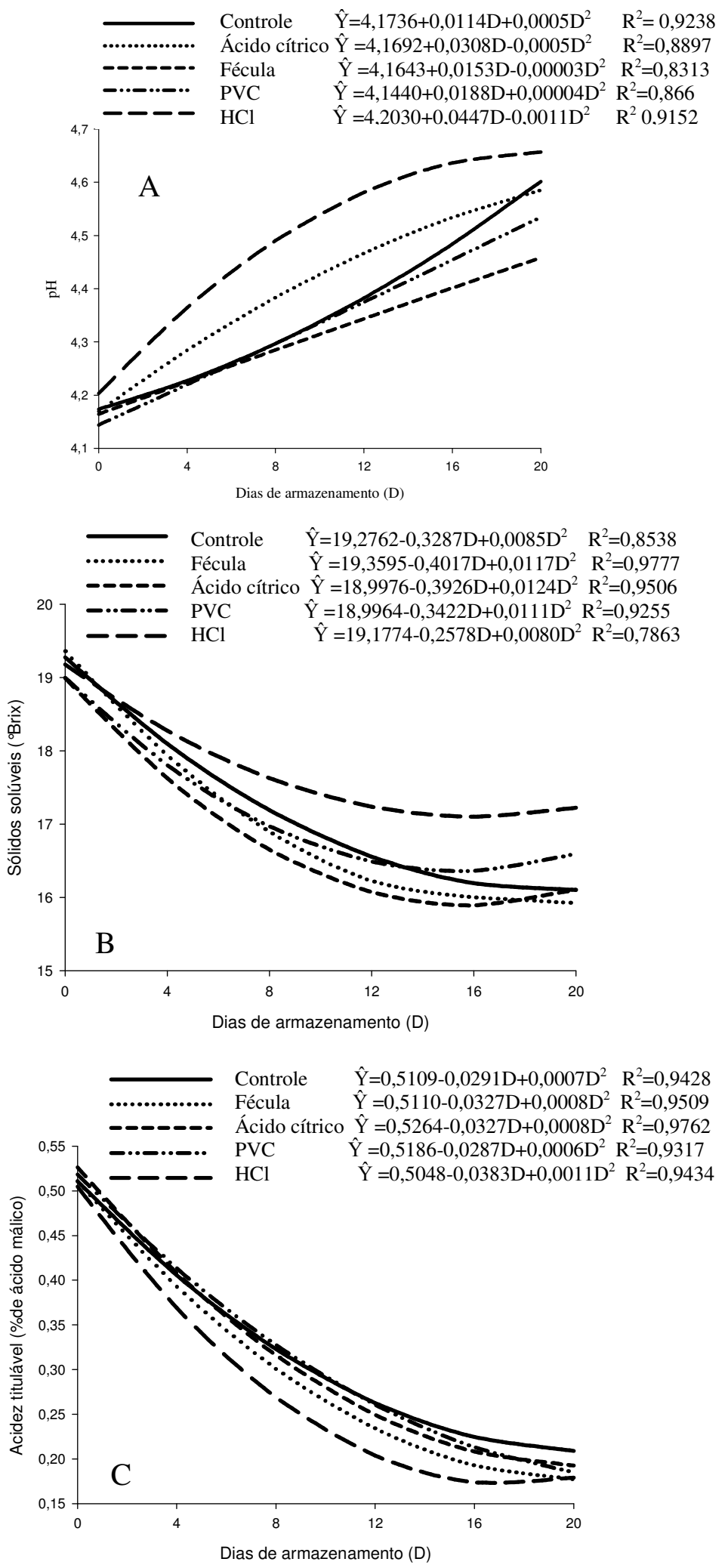

FIGURA 2 - pH (A); teor de sólidos solúveis ( ${ }^{\circ}$ Brix $)(B)$ e acidez titulável (\% de ácido málico) (C) da polpa de frutos de lichieira 'Bengal' armazenados a $5 \pm 1,2{ }^{\circ} \mathrm{C}$ e $90 \pm 5 \%$ de UR, por 20 dias. 

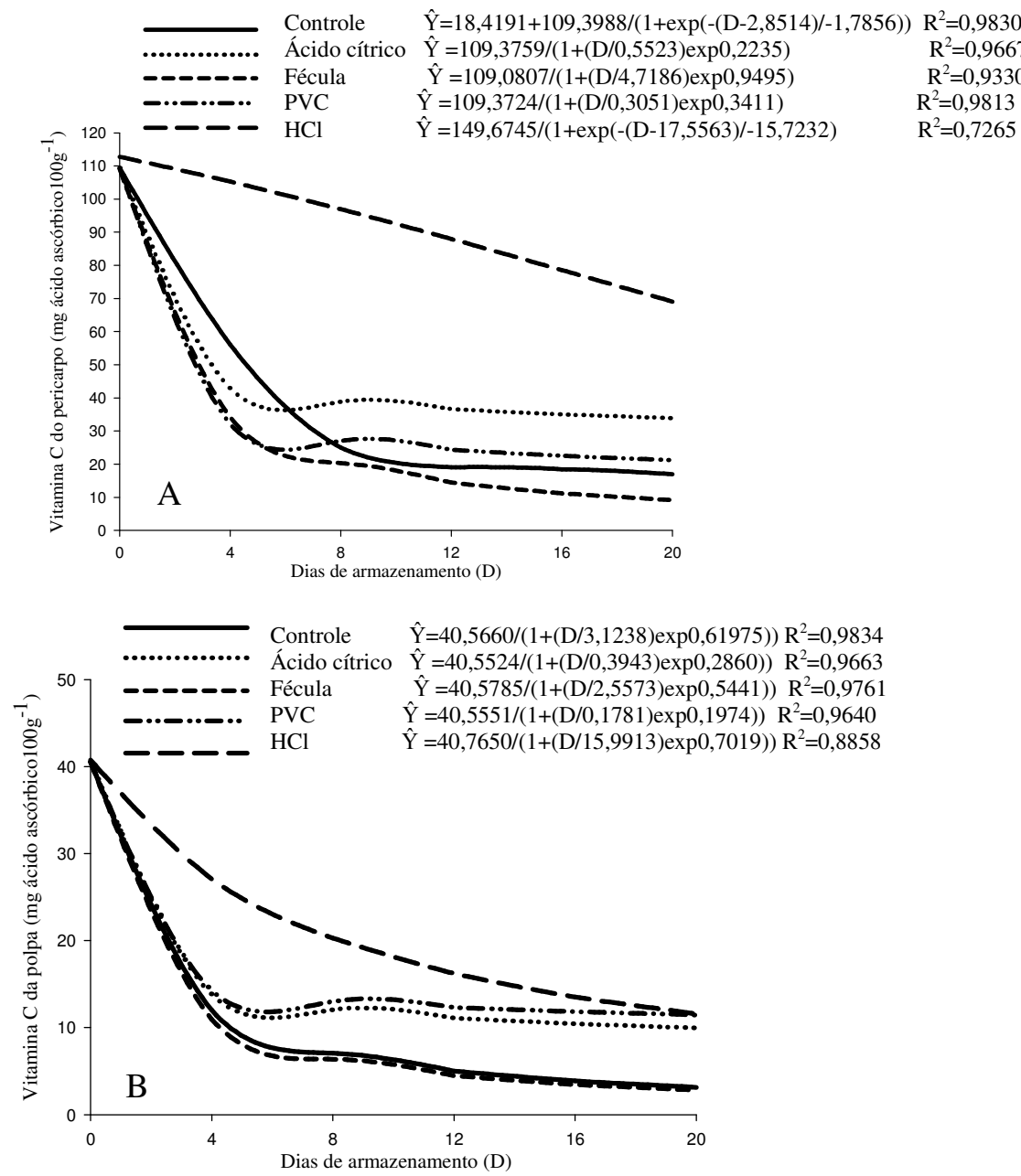

FIGURA 3 - Vitamina C (mg de ácido ascórbico $100 \mathrm{~g}^{-1}$ ) do pericarpo (A) e da polpa (B) de lichias 'Bengal' armazenadas a $5 \pm 1,2{ }^{\circ} \mathrm{C}$ e $90 \pm 5 \%$ de UR, por 20 dias.
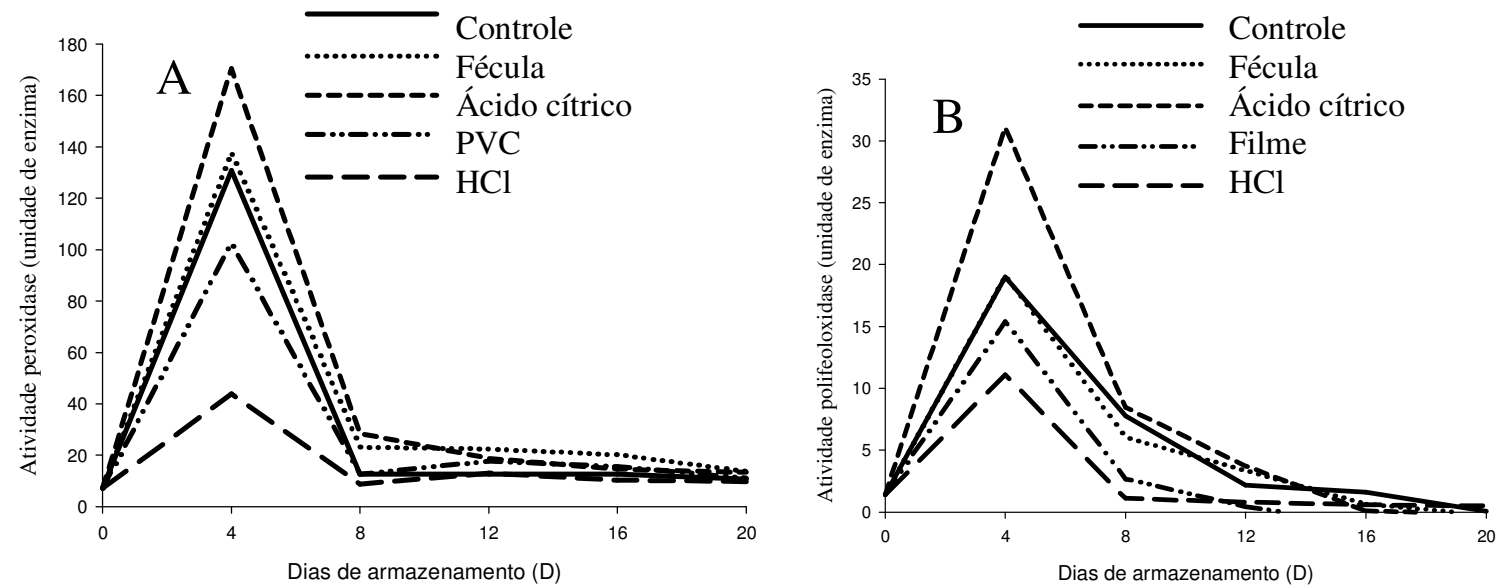

FIGURA 4 - Atividade da peroxidase (A) e da polifenoloxidase (B) do pericarpo de lichias 'Bengal' armazenadas a $5 \pm 1,2{ }^{\circ} \mathrm{C}$ e $90 \pm 5 \%$ de UR, por 20 dias. 


\section{CONCLUSÕES}

1. A utilização da fécula de mandioca para a formação de película protetora, bem como o uso do ácido cítrico não foi eficiente em prevenir ou retardar o escurecimento do pericarpo da lichia.

2. O uso do filme de PVC manteve a coloração vermelha do pericarpo somente até o quarto dia de armazenamento.

3. $\mathrm{O}$ uso do $\mathrm{HCl}$ foi efetivo em prevenir o escurecimento do pericarpo dos frutos durante 20 dias sem comprometer a qualidade pós-colheita.

\section{REFERÊNCIAS}

AOAC. Official methods of analysis of the Association of Official Analytical Chemists International. 16.ed. Washington, 1997. v.2, p.37-10, 42-2, 44-3, 45-16.

CHEN, W.; WU, Z.; SU, M. Postharvest research and handling of litchi in China - a review. Acta Horticulturae, The Hague, n. 558, p. 321-329, 2001.

DUCAMP-COLLIN, M. N.; RAMARSON, H.; LEBRUN, M.; SELF, G.; REYNES, M. Effect of citric acid and chitosan on maintaining red colouration of litchi fruit pericarp. Postharvest Biology and Technology, Amsterdam, v. 49, n. 2, p. 241-246, 2008.

DEL AGUILA, J. S.; HOFMAN, P.; CAMPBELL, T.; MARQUES, J. R.; DEL AGUILA, L. S. H.; KLUGE, R. A. Pré-resfriamento em água de lichia 'B3' mantida em armazenamento refrigerado. Ciência Rural, Santa Maria, v.39, n. 8, p. 2373-2379, 2009.

DELAGUILA, J. S.; HEIFFIG-del A. L.S.; SASAKI, F. F.; ORTEGA, E. M. M.; KLUGE, R. A. Efeito de antioxidantes na taxa respiratória e na produção de etileno de lichia 'Bengal' armazenada sob refrigeração. Revista Iberoamericana de Tecnologia Postcosecha, México, v. 10, p. 8-13, 2009.

DÍAZ-MULA, H.M.; ZAPATA P.J.; GUILLÉN, F.; VALVERDE, J.M.; VALERO, D. M. SERRANO. Modified atmosphere packaging of yellow and purple plum cultivars. 2. Effect on bioactive compounds and antioxidant activity. Postharvest Biology and Technology, Amsterdam, v. 61, p. 110-116, 2011.
FLURKEY, W. H.; JEN, J. J. Peroxidase and polyphenol oxidase activities in developing peachs. Journal of Food Science, Chicago, v. 43, n. 6, p. 1826- 1831, 1978.

HOJO, E. T. D.; DURIGAN, J. F.; YUKI HOJO, R. H.; DONADON, J. R.; MARTINS, R. N. Uso de tratamento hidrotérmico e ácido clorídrico na qualidade de lichia 'Bengal'. Revista Brasileira de Fruticultura, Jaboticabal, v.33, n. 2, p. 10-15, 2011.

JIANG, Y. M.; FU, J.R. Biochemical and physiological changes involved in browning of litchi fruit caused by water loss. Journal of Horticultural Science \& Biotechnology, Ashford, v. 74, n.1, p. 43-46, 1999.

LIMA, R. A. Z.; ABREU, C. M. P. DE; ASMAR, S. A.; CORRÊA, A. D.; SANTOS, C. D dos. Embalagens e recobrimento em lichias (Litchi chinensis sonn.) armazenadas sob condições não controladas. Ciência e Agrotecnologia, Lavras, v. 34, n. 4, p. 914-921, 2010.

MAHAJAN, P. V.; GOSWAMI, T. K. Extended storage life of litchi fruit using controlled atmosphere and low temperature. Journal of Food Processing and Preservation, Westport, v. 28, p. 388-403, 2004.

MAIA, V. M.; Salomão, L. C. C.; Siqueira, D. L.; PUSCHMANN, R.; MOTA FILHO, V. J. G.; CECON, P. R. Tipos e intensidade de danos mecânicos em bananas 'Prata-Anã'; ao longo da cadeia de comercialização. Revista Brasileira de Fruticultura, Jaboticabal, v. 30, p. 365-370, 2008.

McGUIRE, R. G. Reporting of objective color measurements. HortScience, Alexandria, v. 27, n. 12, p. 1254-1260, 1992.

MIZOBUTSI, G. P.; FINGER, F. L.; RIBEIRO, R. A.; PUSCHMANN, R.; NEVES, L. L. de M ; MOTA, W. F. Effect of $\mathrm{pH}$ and temperature on peroxidase and polyphenoloxidase activities of litchi pericarp. Scientia Agrícola, Piracicaba, v.67, n.2, p.213-217, 2010.

NAGAR, P. K. Physiological and biochemical studies during fruit ripening in litchi (Litchi chinensis Sonn.). Postharvest Biology and Technology, Amsterdam, v.4, p. 225-234, 1994. 
NEVES, L. C.; BENEDETTE, R. M.; TOSIN, J. M.; CHAGAS, E. A.; SILVA, V. X. DA; PRILL, M. A. de S.; Roberto, S. R. Produção de blends a partir de frutos tropicais e nativos da Amazônia. Revista Brasileira de Fruticultura, Jaboticabal, v.33, n.1, p. 187-197, 2011.

REUCK, K. DE; SIVAKUMAR, D.; KORSTEN, L. Integrated application of 1-methylcyclopropene and modified atmosphere packaging to improve quality retention of litchi cultivars during storage. Postharvest Biology and Technology, Amsterdam, v.52, n. 1, p.71-77, 2011.

SILVA, D. F. P.; CABRINI, E. C.; ALVES, R. R.; SALOMÃO, L. C. C. Uso do ácido ascórbico no controle do escurecimento do pericarpo de lichia. Revista Brasileira de Fruticultura, Jaboticabal, v. 32, n. 2, p. 618-627, 2010.
ZAUBERMAN, Q. C. et al. Postharvest retention of the red colour of litchi fruit pericarp. Scientia Horticulturae, Amsterdam, v.47, n.1, p.89-97, 1991.

ZHANG, Z.Q.; PANG, X.Q.; YANG, C.; JI, Z.L.; JIANG, Y.M. Purification and structural analysis of anthocyanins from litchi pericarp. Food Chemistry, London, v.84, n.4, p.601-604, 2004.

WATANABE, T.; ROZANE, D. E.; NATALE, W.; FURLAN, C. M.. Avaliação da influência de substâncias fenólicas e carotenoides na anomalia do epicarpo da goiaba, "anelamento". Revista Brasileira de Fruticultura, Jaboticabal, v.33, n.1, p. 8-13, 2011. 